\title{
El impacto del crédito y la cobranza en las utilidades
}

\section{Credit and Collection Profits Impact}

\author{
Juan Manuel Izar Landeta (México) \\ Departamento de Investigación \\ Universidad del Centro de México (UCEM) \\ jmizar@hotmail.com
}

\author{
Carmen B. Ynzunza Cortés (México) \\ Mantenimiento Industrial \\ Universidad Tecnológica de Querétaro \\ ynzunzaber@hotmail.com
}

\section{Resumen}

Este trabajo busca definir la política de crédito y cobranza que le permitan a una pequeña empresa maximizar sus utilidades. El modelo para estimar las utilidades considera el impacto del crédito en el volumen de ventas, así como los costos financieros de las cuentas por cobrar, las cuentas incobrables y los descuentos por pronto pago de los clientes. El crédito para maximizar las utilidades se incrementa si el margen de contribución y el descuento por pronto pago aumentan y si el costo de capital y las cuentas incobrables se reducen, siendo mayor el impacto del margen de contribución y las cuentas incobrables. Con estos hallazgos, se sugiere una apropiada selección de los clientes a los que se vaya a otorgar crédito, de modo que las cuentas incobrables se minimicen, así como dar seguimiento a las cuentas por cobrar, para garantizar que los pagos de los clientes se realicen conforme al plazo establecido.

Palabras Clave: Política de Crédito, Cuentas por Cobrar, Cuentas Incobrables, Descuento por Pronto Pago, Utilidad Máxima

\section{Summary}

This study seeks to define credit and collection policies that allows small businesses to maximize profits. The estimating profit model considers credit on sales volume impact, as well as receivables, uncollectible accounts and prompt payment discounts financial costs. Credit to maximize profits increases if margin of contribution and prompt payment discount also increases, while capital cost and uncollectible accounts are reduced, being greater contribution margin and uncollectible accounts impact. With these findings, an appropriate selection of eligible for credit clients is suggested, so uncollectible accounts are minimized, as well as follow up receivables, to ensure that clients payments are made according to deadline set.

Keywords: Credit Policy, Accounts Receivable, Bad Accounts, Prompt Payment Discount, Maximum Utility
Recibido:

Evaluado:

Aceptado:
17 de enero de 2016

2 de abril de 2017

4 de abril de 2017

\section{PARA CITAR ESTE ARTICULO/TO CITE THIS ARTICLE}

Izar Landeta, J. M. \& Ynzunza Cortés, C. B. (2017).

El Impacto del crédito y la cobranza en las utilidades, Poliantea, 13(24), p. 47-62. 
JUAN MANUEL IZAR LANDETA \& CARMEN BERENICE YNZUNZA CORTÉS 


\section{Introducción}

Es bien sabido en el ámbito comercial que el crédito es una manera de incentivar las ventas, lo que trae consigo más ingresos a las compañías, sin embargo, esto también genera un aumento en las cuentas por cobrar $(C x C)$, así como el riesgo de tener más cuentas incobrables, lo que podría en un momento dado ser contraproducente.

El crédito es, al igual que la publicidad, un factor mercadológico al alcance de las compañías para promover la venta de sus productos, razón por la cual al proceso de aplicar este método en la toma decisiones de crédito, se le denomina la hipótesis de diferenciación del producto.

Por esto es de vital importancia para el bienestar financiero de las empresas definir una política de crédito y cobranza eficientes, que permitan seleccionar a los buenos clientes, que son aquellos que adquieren grandes volúmenes de bienes y los pagan conforme a lo convenido, así como definir un proceso eficiente de cobranza, que permita convertir las $C x C$ en dinero en efectivo para la firma.

El crédito es una forma de venta, ya que los consumidores, especialmente los de clase media o baja, no tienen recursos suficientes para adquirir los bienes de contado, por lo que al brindarles crédito se fomenta el consumo y se estimula la economía.

Una parte muy importante de los activos de las compañías industriales se forma con las $C x C$. En Estados Unidos es una sexta parte (Ross, Westerfield y Jaffe, 2012), mientras que en Gran Bretaña antes del inicio de este tercer milenio era más del 30\% (Asselbergh, 1999). Por tal razón las cuentas por cobrar, como cualquier otro activo de una organización, deben administrarse apropiadamente para que cumplan su función de incrementar las ventas sin que su costo financiero se eleve. Definir una política de crédito no es fácil y ésta afecta al desempeño general de una compañía.

Para establecer la política de crédito, se toman en cuenta 5 factores, que son: los ingresos, los costos, el costo del finan- 
ciamiento del crédito, la probabilidad de falta de pago y los descuentos en efectivo que la firma establezca (Ross et al., 2012).

Algunas empresas pequeñas contratan a terceros - lo que se denomina tercerización o outsourcing-, para llevar a cabo la organización y funciones de crédito.

Antes de otorgar crédito a un nuevo cliente, la empresa debe hacer un análisis cuidadoso con información fidedigna del cliente que le permita decidir si éste es sujeto de crédito, ya que de no hacerlo se corre el riesgo de caer en cuentas incobrables, que vienen a mermar las ganancias.

En Estados Unidos hay compañías como Dun \& Bradstreet, que son especialistas en evaluar el historial crediticio de los clientes.

Otro aspecto que las organizaciones deben tener en cuenta es su política de cobro, que son los procedimientos que establecen para hacer efectiva la cobranza. En esto debe considerarse el costo del proceso, así como la antigüedad de la cuenta que se va a cobrar.

Si la empresa fracasa en sus procedimientos de cobro, aún tiene la opción de utilizar una agencia o empresa de factoraje, quienes suelen tener honorarios elevados por efectuar este tipo de acciones.

En este trabajo se busca definir la mejor política crediticia de una pequeña em- presa que maneja un único producto, incluyendo descuento por pago inmediato, para lo cual se calcula la utilidad anual obtenida para diferentes montos de crédito, a fin de definir la política más conveniente, que será aquella con la cual maximice sus ganancias.

\section{Fundamentos Teóricos}

Las condiciones de crédito que se establezcan en una empresa deben estar en consonancia con las del sector en el que participa, siendo los 2 aspectos determinantes para establecer su límite de crédito los requerimientos de productos que se le proveen y su capacidad de pago.

Los componentes de una política de crédito son básicamente 3 (Ross, et al., 2012):

1. Los términos de venta, que son las condiciones bajo las cuales una empresa realiza la venta de sus productos $y / o$ servicios, que puede ser al contado o a crédito.

2. Análisis de crédito, que es el proceso que la empresa establece para analizar a sus clientes potenciales y determinar quiénes resultan ser sujetos de crédito y quiénes no.

3. La política de cobranza, que establece los mecanismos y procedimientos que la empresa llevará a cabo para realizar el cobro de sus $C x C$.

Los términos de venta se forman con 3 elementos (Ross et al., 2012): (1) el periodo de crédito, (2) los descuentos por pronto pago y (3) los instrumentos de crédito que usan las organizaciones. 
Para el periodo de crédito, la mayoría de las empresas lo establecen entre 30 y 120 días. Los periodos inician usualmente en la fecha de facturación, que por lo regular es la fecha en que se envían los bienes al cliente o se le proporcionan los servicios. Sin embargo hay excepciones, como en el caso de clientes que se encuentran en sitios remotos y por ello reciben los bienes en una fecha posterior, que es la de arranque del periodo de crédito.

El periodo de crédito que establece una organización es también el periodo de pago del cliente y constituye un financiamiento para éste que le permite mejorar su ciclo operativo.

Algunos factores que influyen en la determinación de los periodos de crédito son (Ross et al., 2012): (a) con bienes perecederos, los periodos de crédito serán menores; (b) los artículos con más demanda, tienen periodos más cortos de crédito; (c) los productos baratos, estandarizados y las materias primas suelen tener menores periodos de crédito; (d) con mayor riesgo, menor periodo de crédito; (e) a menores montos de las cuentas, los periodos de crédito serán más cortos; (f) con más competencia, los periodos de crédito serán mayores; $\mathrm{y}$ (g) los periodos de crédito dependen del tipo de cliente.

Los descuentos por pronto pago son cantidades que la firma acuerda rebajar al cliente si paga en un periodo corto. A las empresas les permite recuperar su cartera rápidamente, así como reducir el monto de $C x C$, mientras que para el cliente el descuento significa un ahorro que podría ser atractivo.

Los instrumentos de crédito son los medios con los cuales se deja evidencia de haber contraído un crédito (Ross et al., 2012), siendo el más usual la factura, la que se envía al cliente junto con los bienes, en su caso éste la firma dando su aceptación, con lo cual se establece la cuenta por cobrar. Otro instrumento es el pagaré, que se utiliza en ocasiones especiales, cuando el monto de deuda es elevado, si no se tiene suficiente confianza con el cliente o se anticipa que podría haber problemas para el cobro.

Leitch y Lamminmaki (2011) comentan que dos métricas ampliamente usadas para evaluar el desempeño en el manejo de las $C x C$ han sido el periodo promedio de cobro y su antigüedad. Señalan que estas métricas son deficientes, especialmente cuando se utilizan con propósitos de evaluación interna. Ante esto proponen que para la antigüedad de las cuentas se relacione su monto con su valor original que dio lugar al crédito y para el periodo promedio de cobro, el monto de las $C x C$ se divida en categorías según su antigüedad, la que debe corresponder a las ventas a crédito que las generaron.

En un estudio con 77 empresas iraníes, Azam (2012) aplicó un modelo de regresión logística del riesgo en el manejo de las $C x C$, basado en 4 variables: la discriminación de precios, la tasa de deuda, el crecimiento de la firma y la tasa corriente, encontrando que si el valor de las $C x C$ llega a $25 \%$ o más de reclama- 
ciones, se requerirán colaterales u otros instrumentos de garantía.

Stanford (1995) ha aplicado un proceso markoviano de decisión de 4 etapas al manejo de las $C x C$, en el cual encuentra que ni las cuentas incobrables ni los ingresos por intereses son muy sensibles a cambios en las probabilidades de transición, pero algunas de estas probabilidades impactan más que otras. Además también encontró que un porcentaje de aumento dado en la tasa de incremento de nuevos cargos produce aproximadamente una tasa igual de disminución de las cuentas incobrables.

Asselbergh (1999) analiza el manejo de las $C x C$ bajo dos puntos de vista: el estratégico, que consiste en ver el crédito como un aspecto ventajoso para el proveedor, que necesita flexibilidad en el manejo de las $C x C$; y el del manejo del riesgo, que se basa en los principios relacionados con el riesgo moral. La perspectiva estratégica explica la decisión de externalizar el manejo de las $C x C$, mientras que no tiene poder predictivo en cuanto al manejo del riesgo. Además no encontró relación entre el periodo promedio de cobro ni la obtención de economías de escala con la posibilidad de externalizar el manejo de las $C x C$. Las empresas que usan el crédito como un factor de fijación de precios y las de mayor edad y con mayores estructuras de activos, tienden al manejo interno de sus $C x C$.

Ben-Horim y Levy (1983) afirman que si una empresa desea mantener los beneficios que ofrece al comprador, los descuentos por pronto pago deben aumen- tar de acuerdo con las tasas de inflación, mientras que el comprador, por su parte, es más probable que haga pagos anticipados, aun si el beneficio es menor que en una situación en la que no haya riesgo de inflación.

Kontuš (2013) propone un modelo matemático para calcular los ahorros netos y establecer la política crediticia, de modo que se obtenga una rentabilidad neta máxima, ya que el manejo de las $C x C$ impacta a la rentabilidad del negocio. En un estudio con compañías croatas encuentra que si las $C x C$ aumentan, la rentabilidad también lo hace, por lo cual recomienda la implementación de una política de crédito apropiada.

Hyndman y Serio (2006) afirman que en caso de que la competencia en Indonesia se agudice, las organizaciones tenderán a dar menos crédito para incrementar sus ventas.

Nguyen (2011) en un estudio con 260 empresas vietnamitas encuentra que proveer crédito comercial a las empresas tiene un impacto significativo y positivo para incrementar el volumen de ventas $y$ dicho impacto es mayor en las empresas jóvenes.

Blazenko y Vandezande (2003) hacen un análisis similar incluyendo el costo de las cuentas incobrables y llegan a la conclusión que el crédito se asocia con el margen de beneficio y esta relación puede ser positiva o negativa dependiendo de la elasticidad de la demanda y la intensidad de capital del sector. En caso que la elasticidad sea muy alta, la 
relación entre el crédito y el margen de beneficio es más bien negativa. La razón óptima de crédito a ingresos es directamente proporcional a la elasticidad de la demanda respecto al crédito e inversamente proporcional a la elasticidad del precio.

Dinca y Bociu (2015) han aplicado análisis discriminante en Rumania para predecir cuáles empresas pueden llegar a la quiebra, siendo un mecanismo de apoyo para las instituciones financieras, a fin de conocer quiénes son sujetos de crédito.

Johnson, Pence y Vine (2014) aplicaron un modelo de auto regresión al caso de la venta de autos en Estados Unidos, la cual cayó en 20\% durante la recesión de 2007 a 2009, mientras que el número de créditos para adquirirlos disminuyó $33 \%$. Con el modelo determinaron que las condiciones de crédito influyen significativamente en la venta de autos.

Lieber y Orgler (1975) aplicaron un modelo integrado para el manejo de las $C x C$, el cual toma en cuenta los descuentos por pronto pago, el periodo de crédito, los cargos por pagos tardíos, los costos de cobranza, las cuentas incobrables y los periodos de cobro de cuentas vencidas, de modo que se alcance la ganancia máxima.

Behner, Perez, Smith y Varuolo (2015), han estudiado la relación entre el crédito y las ventas, considerando que ambas áreas pueden proveer información valiosa de las condiciones de crédito de clientes potenciales y el establecimiento de políticas crediticias apropiadas para atraer nuevos clientes, por lo cual enfatizan la importancia de que los altos ejecutivos de la organización prioricen la buena relación entre ambas funciones.

Mao y Sarndal (1974) señalan que un aspecto crítico del manejo del crédito es el control del riesgo de las cuentas incobrables. Proponen para este fin medir la varianza de la tasa de cuentas perdidas respecto a ventas bajo varios supuestos relacionados al número y distribución de tamaño de los clientes. Las mejores predicciones se han logrado en el caso de que el tamaño de la empresa del cliente sea pequeño y cuando la distribución de ventas es amplia entre los clientes. Los pronósticos no han sido acertados cuando hay correlación entre el número y tamaño de los clientes, como en el caso de que las ventas se realicen con pocos clientes de gran tamaño.

Michalski (2008) propone el manejo de las $C x C$ mediante el uso de la teoría de manejo del portafolio para la toma de decisiones respecto a qué clientes dar crédito. Un incremento de las $C x C$ aumenta el capital de trabajo y los costos de su mantenimiento y manejo, los que hacen disminuir el valor de la firma. Sin embargo, una buena política de administración de las $C x C$, aunada al buen manejo del portafolio, puede incrementar el valor de la firma.

Las decisiones del crédito comercial dependen de factores como el grado de competencia del mercado, el tipo de bienes y servicios que se ofertan, la estacionalidad y elasticidad de la demanda, los precios, el tipo de cliente y los márgenes de beneficio de la firma. 


\section{Metodología}

El objetivo de este trabajo es encontrar la política de crédito que permita maximizar las utilidades, para lo cual se considera que un mayor monto de crédito incrementa el volumen de ventas, pero hay el riesgo de caer en cuentas incobrables, además deben considerarse el costo financiero de las $C x C$ y los descuentos que se hagan por pronto pago.

Para estas partidas, los ingresos de ventas se componen del producto del precio unitario de cada artículo, multiplicado por el volumen vendido, conforme a la ecuación (1):

$$
I=P V
$$

Donde:

$I=$ Ingresos por ventas, $\$ /$ mes

$P=$ Precio unitario del producto, $\$$ / unidad

$V=$ Volumen vendido de artículos, unidades / mes

Mientras que las cuentas por cobrar $(C x C)$ pueden obtenerse con la siguiente relación:

$$
C \times C=I C r \frac{P P C}{365}
$$

Siendo $C r$ la fracción del total de ingresos obtenidos mediante crédito y $P P C$ el periodo promedio de cobro, que para el caso de la empresa en cuestión, dado que la opción del pronto pago es hacerlo de inmediato, o bien con descuento en un periodo dado, denominado Per, puede estimarse con la fórmula siguiente:

$$
P P C=C r P e r
$$

Por su parte el costo financiero $C_{f}$ de las cuentas por cobrar, se obtiene con la ecuación siguiente:

$$
C_{f}=C x C k_{0}
$$

Siendo $k_{0}$ el costo de capital de la organización.

Por su parte el costo de las cuentas incobrables $C_{\text {Inc }}$ evaluado no al precio de venta, sino al costo variable unitario $(C V)$, viene dado por la expresión siguiente:

$$
C_{\text {Inc }}=\operatorname{CrIncVCV}
$$

Siendo Inc la fracción del monto incobrable del crédito otorgado a la clientela.

El monto de descuento $C_{D c t}$ aprovechado por aquellos clientes que pagan de inmediato también viene a disminuir la utilidad alcanzada. Su monto puede estimarse con la ecuación (6):

$$
C_{D c t}=P V(1-C r) D c t
$$

Siendo Dct el porcentaje de descuento por pronto pago que se da a los consumidores.

La utilidad de la empresa Ut se forma con la utilidad bruta, a la que deben descontarse los costos antes mencionados, para llegar a la relación dada por la fórmula siguiente:

$$
\begin{array}{r}
U t=(P-C V) V-C F \\
-C x C k_{0}-C r I n c V C V \\
\quad-P V(1-C r) D c t
\end{array}
$$

Donde el único término que no se ha definido previamente son los costos fijos $C F$, expresados en forma mensual. 
El margen de contribución $M C$ viene dado por la expresión siguiente:

$$
M C=\frac{P-C V}{P}
$$

Entonces se trata de definir las variables como el descuento por pronto pago, el costo de capital, la fracción de cuentas incobrables y el margen de contribución que lleven a una política óptima de crédito.

\section{Aplicación a un caso empírico}

El caso de la pequeña empresa bajo análisis es que maneja un único producto, otorga $4 \%$ de descuento por pago inmediato a sus clientes, o bien el pago sin descuento a 40 días. Su producto lo vende a un precio unitario de $\$ 850$, su costo variable es $\$ 480$ por artículo y tiene costos fijos por $\$ 2$ millones anuales, su costo de capital es $12 \%$ anual y su volumen de ventas promedio es de 12,000 artículos por año para un monto de crédito del $80 \%$ del total de las ventas. Las cuentas incobrables han sido en promedio $6 \%$ del monto de las cuentas por cobrar. Además la empresa ha obtenido empíricamente de experiencias anteriores la siguiente relación entre el volumen de ventas y el monto de crédito otorgado a sus clientes:

Tabla 1. Cambio en el volumen de ventas en función del crédito otorgado

\begin{tabular}{|c|c|}
\hline Crédito \% & Cambio en el volumen de ventas \% \\
\hline \hline 0 & -30 \\
\hline 20 & -20 \\
\hline 50 & -10 \\
\hline 80 & 0 \\
\hline 100 & 12 \\
\hline \multicolumn{2}{|c|}{ Fuente: Elaboración propia } \\
\hline
\end{tabular}

Con base en esta información la empresa desea establecer una política de crédito que maximice sus ganancias.

Para esto, lo primero es establecer la relación entre el monto de crédito y el cambio en el volumen de ventas, el cual se muestra gráficamente en la figura 1 , siendo la relación entre las variables lineal y con un ajuste muy bueno, que permite utilizar la ecuación obtenida para estimar el cambio en el volumen de ventas $(\Delta V)$ en función del crédito otorgado a los clientes. Esta ecuación es:

$$
\Delta V=0.3971 C r-0.2945
$$

Ya con este cambio se ajusta el volumen de ventas y se obtiene la utilidad respectiva. Así para los datos del caso base, si la empresa otorga el $80 \%$ de crédito, el cambio en el volumen de ventas estimado con la ecuación (9) es:

$$
\begin{aligned}
\Delta V & =0.3971(0.80)-0.2945 \\
& =0.02318
\end{aligned}
$$

$Y$ por tanto la venta sería de $(12,000)(1.02318)=12,278$ artículos anuales.

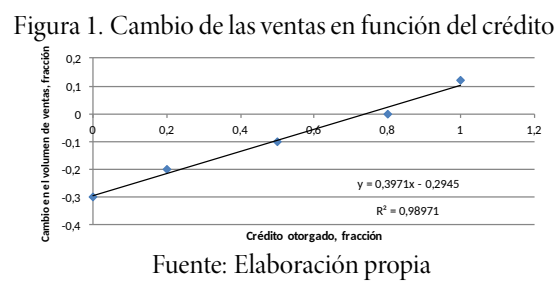

El margen de contribución de la empresa puede calcularse con la ecuación (8):

$$
M C=\frac{850-480}{850}=0.435
$$


Con el volumen de ventas estimado, los ingresos serán por un monto de $\$ 10,436,436$ anuales y el periodo promedio de pago obtenido con la ecuación (3) es:

$$
P P C=(0.80)(40)=32 \text { días }
$$

Con esto, el monto de las cuentas por cobrar se obtiene con la fórmula (2):

$$
\begin{aligned}
C x C & =(10,436,436)(0.80) \frac{32}{365} \\
& =\$ 731,980.17
\end{aligned}
$$

Y su costo financiero con la ecuación (4):

$$
\begin{aligned}
C_{f} & =(731,980.17)(0.12) \\
& =87,837.62 \text { \$/año }
\end{aligned}
$$

Por su parte el costo del incobrable se estima con la ecuación (5):

$$
\begin{aligned}
C_{\text {Inc }} & =(0.80)(0.06)(12,278)(480) \\
& =282,888.81 \text { \$/año }
\end{aligned}
$$

El monto del descuento otorgado a los clientes que pagan de inmediato se calcula con la ecuación (6):

$$
\begin{aligned}
C_{\text {Dat }} & =(850)(12,278)(1-0.80)(0.04) \\
& =83,491.49 \text { \$/año }
\end{aligned}
$$

Siendo la utilidad:

$$
\begin{array}{r}
U t=(850-480)(12,78)-2,000,000 \\
-87,837.62-282,888.81-83,491.49 \\
=2,088,701.29 \$ \text { /año }
\end{array}
$$

Si se hacen cálculos similares para otros montos de crédito otorgado a los clientes, la máxima ganancia se logra con el 100\% de crédito, resultando en un monto de 2,366,586 \$/año.
Uno de los objetivos del presente trabajo es analizar el impacto de las diferentes variables sobre el crédito a establecer para alcanzar la máxima utilidad, lo cual se plantea variando los valores de variables como el costo de capital, al que se han asignado 3 diferentes valores, el del caso base de $12 \%$ anual, más $0 \%$ y $25 \%$; el descuento por pronto pago, que además del valor del caso base de $4 \%$, se han establecido valores del 0 y $8 \%$; el monto de incobrables, al que se han asignado valores de 2, 12, 16 y 20\%, además del valor del caso base del 6\%; y el margen de contribución, al que además del caso base de 0.435 , se han dado valores de $0.467,0.412,0.353,0.294$ y 0.235 . Esto hace un total de $3 \times 3 \times 5 \times 6=$ 270 combinaciones, cuyos resultados se han analizado mediante regresión lineal múltiple para definir el impacto de cada variable sobre el crédito requerido para maximizar las ganancias.

Al hacer el análisis de regresión con el monto de crédito para lograr la máxima utilidad como variable dependiente y con el costo de capital, el monto de cuentas incobrables, el descuento otorgado por pronto pago y el margen de contribución como variables independientes, se dan los resultados que ahora se presentan.

Todas las variables independientes han tenido correlación significativa con el crédito, al nivel de significancia de 0.01 .

El coeficiente de determinación ajustado ha sido 0.57 , lo que hace ver que la ecuación de regresión da cuenta del 57\% de la varianza del crédito. 
La ecuación obtenida con la regresión es:

$$
\begin{aligned}
C r & =0.42-0.367 k_{0}-2.026 \text { Inc } \\
& +1.993 D c t+1.73 M C \quad(10)
\end{aligned}
$$

La tabla 2 presenta los valores de los coeficientes beta estandarizados y su significancia estadística para las variables independientes:

Tabla 2. Valores de betas estandarizadas y su significancia estadística

\begin{tabular}{|c|c|c|}
\hline $\begin{array}{c}\text { Variable } \\
\text { Independiente }\end{array}$ & $\begin{array}{c}\text { Beta } \\
\text { Estadarizada }\end{array}$ & Significancia \\
\hline \hline$K_{0}$ & -0.137 & 0.001 \\
\hline$I n c$ & -0.484 & 0.000 \\
\hline$D c t$ & 0.238 & 0.000 \\
\hline$M C$ & 0.515 & 0.000 \\
\hline
\end{tabular}

Fuente: Elaboración propia

Todas las variables han resultado significativas, siendo las de mayor impacto el margen de contribución $(M C)$ en sentido directo y la fracción de cuentas incobrables (Inc) en sentido inverso.

El costo de capital y las cuentas incobrables se han relacionado de manera inversa con el crédito, puesto que han resultado con betas negativas; mientras que el descuento por pronto pago y el margen de contribución lo han hecho en forma directa, al tener betas positivas.

No ha habido problema de colinearidad entre las variables independientes, puesto que todos los índices de condición de las variables han resultado menores de 15 , pero sí en cuanto a la independencia de los residuos, ya que el coeficiente de Durbin Watson ha sido 0.91 , lo que indica que éstos se correla- cionan positivamente y no son independientes.

La prueba de normalidad de los residuos se muestra en la figura 2, en la cual puede verse que no hay problema con este supuesto de la técnica de regresión.

Figura 2. Gráfico de los residuos estandarizados

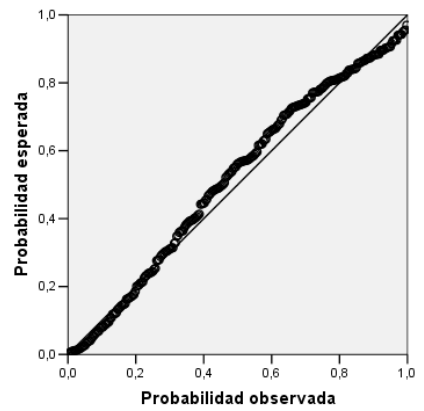

Fuente: Elaboración propia

De los 270 cálculos realizados, se observó que si el incobrable se ubica en su valor mínimo (2\%), el monto de crédito para maximizar las ganancias es del $100 \%$, independientemente de los valores que adopten las demás variables, como son el costo de capital, el margen de contribución y el descuento por pronto pago.

Además si el margen se ubica en cualquiera de sus dos valores máximos (0.467 y 0.435) el crédito a otorgar para lograr máxima utilidad es 100\% para cualquier valor del costo de capital y el descuento, pero siempre y cuando las cuentas incobrables no rebasen el 12\% del total del crédito.

Asimismo se ha realizado análisis de regresión múltiple con las mismas 
variables independientes, pero considerando la utilidad como variable dependiente.

En este caso el descuento por pronto pago no ha sido significativo, y el coeficiente de determinación ajustado fue de 0.984 , lo que hace un ajuste excelente que explica más del $98 \%$ de la varianza de la utilidad.

Al hacer análisis de sensibilidad de la utilidad lograda con cada variable significativa, como han sido el margen de contribución, el monto de cuentas incobrables y el costo de capital, se han obtenido los resultados siguientes.

Figura 3. Variaciónd de la utilidad con el margen de contribución

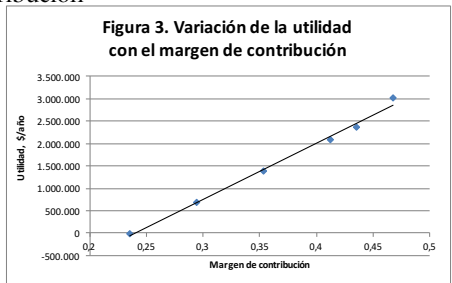

Fuente: Elaboración propia

En cuanto al margen de contribución, si se mantienen las demás variables en sus valores del caso base y se modifica el margen para ver su impacto en la utilidad, se obtiene el gráfico de la figura 3.

El ajuste es lineal con un valor del coeficiente de determinación ajustado de 0.9914 y una pendiente de la línea recta de $12,611,477$, que indica que por cada 0.01 unidades que aumente el margen de contribución, la utilidad sube 126,115 pesos.
Si se hace lo mismo con el monto de cuentas incobrables, se obtiene la figura 4 , la que muestra que el ajuste lineal ha sido perfecto, ya que el coeficiente de determinación ha sido 1.00 y la pendiente de la recta resultó en $-6,350,976$, lo que indica que por cada $1 \%$ que aumenten las cuentas incobrables, la utilidad disminuye en $\$ 63,510$ anuales.

Figura 4. Cambio de la utilidad con el monto de cuentas incobrables

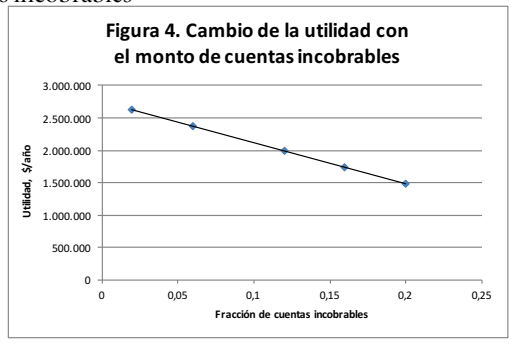

Fuente: Elaboración propia

Al hacer el análisis de sensibilidad de la utilidad con el costo de capital, se obtiene el gráfico de la figura 5, en el cual se aprecia que el ajuste lineal también ha sido perfecto, ya que el valor del R2 ha sido 1.00 y la pendiente de la recta es $-1,232,495$, lo que indica que por cada punto porcentual que aumente el costo de capital, la utilidad disminuye en un monto de $\$ 12,325$ anuales.

Figura 5. Cambio de la utilidad con el costo de capital

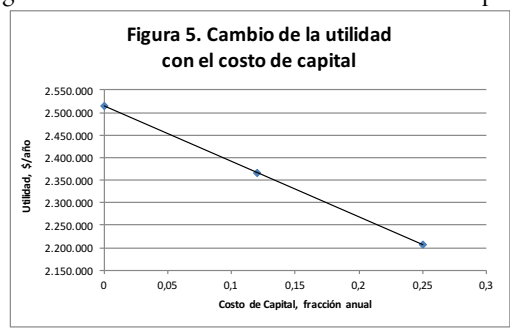

Fuente: Elaboración propia 
Finalmente se presenta en la figura 6 la variación del monto de crédito para lograr la máxima utilidad en función del cambio en el margen de contribución para el caso en que el costo de capital sea del $25 \%$ anual, el monto de cuentas incobrables del $20 \%$ y el descuento por pago inmediato del $4 \%$, ya que en estas condiciones la política de otorgar 100\% de crédito no siempre es la que produce la máxima utilidad, tal como sucedía con valores bajos del costo de capital y el monto de cuentas incobrables.

Figura 6. Cambio de la utilidad con el costo de capital

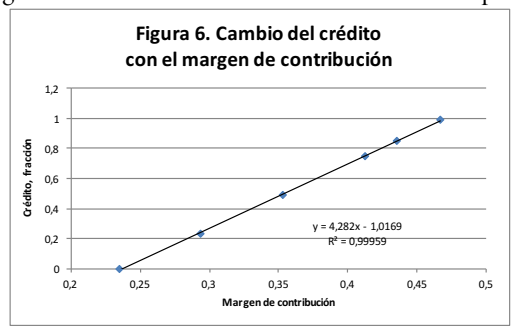

Fuente: Elaboración propia

El ajuste lineal ha sido excelente y la relación de cambio es que por cada $1 \%$ que el margen de contribución se incremente, el crédito para alcanzar la utilidad máxima aumenta $4.28 \%$. Vale la pena comentar que esta relación de cambio se ve afectada si se utilizan otros valores de las variables implicadas como el descuento, más no el sentido de la relación, el cual se mantiene: con más margen, el crédito a otorgar para maximizar las ganancias aumenta.

\section{Conclusiones}

Como era de esperarse para el caso de la empresa bajo análisis, el monto de crédito para maximizar las ganancias se incrementa en caso que el margen de contribución y el descuento por pronto pago aumenten, igualmente si el costo de capital y las cuentas incobrables se reducen, siendo mayor el impacto del margen de contribución y el de las cuentas incobrables.

Se confirma que las ventas se ven afectadas por las condiciones de crédito de la firma, tal y como lo han afirmado varios autores referenciados en el marco teórico, como Nguyen (2011) y Johnson et al (2014).

Para el caso de la utilidad, ésta no se ve afectada por el descuento por pronto pago, ya que en la mayoría de los 270 casos, el monto de crédito se ubicó en el $100 \%$ y en estas condiciones no hay descuento. Sin embargo, las ganancias aumentan directamente con el margen de contribución e inversamente con las cuentas incobrables y el costo de capital, siendo mayor el impacto del margen y los incobrables.

Por esto, establecer una política de crédito que incluya el análisis de los clientes para determinar si son susceptibles de crédito y dar el seguimiento a las cuentas por cobrar para no tener un monto elevado de cuentas incobrables, es imprescindible para optimizar los resultados financieros de la empresa bajo análisis, tal y como lo afirman Michalski (2008) y Kontuš (2013).

Asimismo el hecho de tener mayores márgenes de contribución de los productos permite otorgar crédito, ya que al aumentar el volumen de bienes ven- 
didos, la probabilidad de ganar con las ventas adicionales es mayor que la de perder con las cuentas incobrables que se puedan generar, lo que se ha confirmado en este estudio, a diferencia de lo que señalan Blazenko y Vandezande (2003), que esto depende de la elasticidad de la demanda. No obstante, el margen de contribución es una variable que depende básicamente de las condiciones del mercado y la eficiencia operativa de las empresas, no directamente del manejo del crédito y las cuentas por cobrar.

Por su parte el costo de capital es una variable que se establece dependiendo de la forma como se financia una empresa y las fuentes de financiamiento que utiliza, no directamente de su política crediticia.

Además deben analizarse otras opciones de establecer descuentos por pronto pago a los clientes y no dejar como única alternativa la que se tiene actualmente del pago inmediato con descuento del $4 \%$, o el pago total del monto facturado a su plazo de vencimiento de 40 días, lo que implicaría flexibilizar la política crediticia de la firma, aspecto que ya no se ha considerado en este trabajo, pero podría abordarse posteriormente.

\section{Referencius}

Asselbergh, G., (1999). A Strategic Approach on Organizing Accounts Receivable Management: Some Empirical Evidence. Journal of Management and Governance, 3 , 1-29.
Azam, J., (2012). The Determinant Factors in Presenting a Model of Accouns Receivables Risk Management: Iranian case. International Journal of Information, Business and Management, 4(3), 99-111.

Behner, T., Perez, S., Smith, B., \& Varuolo, S., (2015). The Dynamic Duo: Credit and Sales: A Look at Relationship Best Practices. Business Credit, 6, 38-40.

Ben-Horim, M., \& Levy H., (1983). Management of Accounts Receivable under Inflation. Financial Management, 1, 42-48.

Blazenko, G. W., \& Vandezande, K., (2003). The Product Differentiation Hypothesis for Corporate Trade Credit. Managerial Decision Economics, 24(6-7), 457469.

Dinca, G., y Bociu, M., (2015). Using discriminant analysis for credit decision. Bulletin of The Transilvania University of Brasov, Series V: Economic Sciences, 8(2), 277288.

Hyndman, K., y Serio, G., (2006). Competition and Inter-firm Credit Theory and Evidence from Firm-level data in Indonesia. Journal of Development Economics, 93, 88-108.

Johnson, K., Pence, K., \& Vine, D., (2014). Auto Sales and Credit Supply. Board of Governors of the Federal Reserve System, 1-40. 
Kontuš, E., (2013). Management of Accounts Receivable in a Company. Ekonomska Misao I Praksa, 22 (1), 21-38.

Leitch, P., \& Lamminmaki, D., (2011). Refining Measures to Improve Performance Measurement of the Accounts Receivable Collection Function. Journal of Applied Management Accounting Research, 9(2), 1-20.

Lieber, Z., \& Orgler, Y. E., (1975). An Integrated Model for Accounts Receivable Management. Management Science, 22(2), 212-219.

Mao, J. C. T., \& Sarndal, C. E., (1974). Controlling Risk in Accounts Receivable Management. Journal of Business Finance \& Accounting, 1(3), 395-403.
Michalski, G., (2008). A Portfolio Management Approach in Accounts Receivable Management. South East European Journal of Economics \& Business, 3(2), 8995.

Nguyen, L. T. U., (2011). Trade Credit in The Rice Market of The Mekong Delta in Vietnam, Tesis Doctoral, Universidad de Groningen, Holanda, 148p.

Ross, S. A., Westerfield, R. W., \& Jaffe, J., (2012). Finanzas Corporativas, 9a edición, Mc Graw Hill: México.

Stanford, R. E., (1995). A Structured Sensitivity Analysis for a Markov Model of Accounts Receivable. Journal of Accounting, Auditing \& Finance, 10(3), 643-653. 
JUAN MANUEL IZAR LANDETA \& CARMEN BERENICE YNZUNZA CORTÉS 